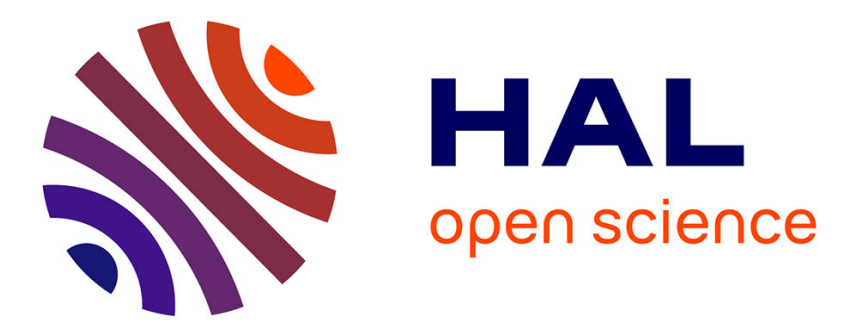

\title{
Three-weight codes, triple sum sets, and strongly walk regular graphs
}

Patrick Sole, Minjia Shi

\section{To cite this version:}

Patrick Sole, Minjia Shi. Three-weight codes, triple sum sets, and strongly walk regular graphs. Designs, Codes and Cryptography, 2019, 87, pp.2395 - 2404. 10.1007/s10623-019-00628-7 . hal02411038

\section{HAL Id: hal-02411038 \\ https://hal.inria.fr/hal-02411038}

Submitted on 17 Dec 2019

HAL is a multi-disciplinary open access archive for the deposit and dissemination of scientific research documents, whether they are published or not. The documents may come from teaching and research institutions in France or abroad, or from public or private research centers.
L'archive ouverte pluridisciplinaire HAL, est destinée au dépôt et à la diffusion de documents scientifiques de niveau recherche, publiés ou non, émanant des établissements d'enseignement et de recherche français ou étrangers, des laboratoires publics ou privés. 


\title{
Three-weight codes, triple sum sets, and strongly walk regular graphs
}

\author{
Minjia Shi ${ }^{1} \cdot$ Patrick Solé ${ }^{2}$
}

Received: 5 November 2018 / Revised: 3 March 2019 / Accepted: 6 March 2019 / Published online: 16 March 2019 (c) Springer Science+Business Media, LLC, part of Springer Nature 2019

\begin{abstract}
We construct strongly walk-regular graphs as coset graphs of the duals of three-weight codes over $\mathbb{F}_{q}$. The columns of the check matrix of the code form a triple sum set, a natural generalization of partial difference sets. Many infinite families of such graphs are constructed from cyclic codes, Boolean functions, and trace codes over fields and rings. Classification in short code lengths is made for $q=2,3,4$.
\end{abstract}

Keywords Strongly walk-regular graphs $\cdot$ Three-weight codes $\cdot$ Triple sum sets

Mathematics Subject Classification Primary 05 E $30 \cdot$ Secondary 94 B 05

\section{Introduction}

Since the seminal article of Delsarte [6], there is a well-known interplay between two-weight codes and strongly regular graphs (SRG) via the coset graph of the dual code $[1,2,19]$. Strongly walk-regular graphs (SWRG) were introduced in [20] as a generalization of strongly regular graphs. Instead of a regularity condition bearing on paths of length 2, the notion of SWRG demands a regularity on paths of length $\ell>1$. Specifically, a graph is $\ell-S W R G$ if there are three integers $(\lambda, \mu, v)$ such that the number of paths of length $\ell$ between any two vertices $x$ and $y$ is

- $\lambda$ if $x$ and $y$ are connected;

- $\mu$ if $x$ and $y$ are disconnected;

- $v$ if $x=y$.

\section{Communicated by J. H. Koolen.}

This research is supported by National Natural Science Foundation of China (61672036), Excellent Youth Foundation of Natural Science Foundation of Anhui Province (1808085J20).

Minjia Shi

smjwcl.good@163.com

1 School of Mathematical Sciences of Anhui University, Anhui 230601, People's Republic of China

2 Aix Marseille Univ, CNRS, Centrale Marseille, I2M, Marseille, France 
Thus $\ell=2$ is the case of SRGs. Note that, by definition SRGs are $\ell$-SWRG for all $\ell>1$. In [4] was introduced the notion of triple sum set, a natural generalization of partial difference sets, which enter the study of two-weight codes [3,14]. Let $\mathbb{F}_{q}$ denote the finite field of order $q$. The set $\Omega \subseteq \mathbb{F}_{q}^{k}$ is a triple sum set (TSS) if it is stable by scalar multiplication and if there are constants $\sigma_{0}$ and $\sigma_{1}$ such that a nonzero $h \in \mathbb{F}_{q}^{k}$ can be written as

$$
h=x+y+z, \text { with } x, y, z \in \Omega,
$$

- $\sigma_{0}$ times if $h \in \Omega$,

- $\sigma_{1}$ times if $h \in \mathbb{F}_{q}^{k} \backslash \Omega$.

This was generalized in $[11,12]$ to the notion of $s$-sum set by replacing the equation $h=$ $x+y+z$ by $h=\sum_{i=1}^{s} x_{i}$ where each $x_{i} \in \Omega$.

The aim of this note is to connect these two notions by means of a third one: the coset graph of a projective code [1]. The graphical approach of [20] makes several results on multiple sum sets of $[4,11,12]$ more transparent. Sometimes, new results are obtained (Theorem 6). In the other direction, three-weight codes with known weight distributions provide many infinite families of SWRGs with integral spectra that would be hard to construct otherwise. Some classical tools of coding theory (Pless power moments, MacWilliams transform) allow us to classify short codes over $\mathbb{F}_{q}$ with $q=2,3,4$, that satisfy the hypotheses of Theorem 6 , thus yielding $s$-sum sets for all odd $s>1$.

The material is organized as follows. The next section collects the necessary definitions, notations, and background material. The theoretical results are contained in Sect. 3. Section 4 classifies the three-weight codes of short length with the parameters of Theorem 6 for $q=$ $2,3,4$. Sect. 5 is devoted to many examples of constructions. Section 6 puts the results into perspective and lists several challenging open problems.

\section{Definitions and notation}

\subsection{Graphs}

All graphs in this note are finite, undirected, connected, without multiple edges. Loops, however, will be allowed. The neighborhood $\Gamma(x)$ is the set of vertices connected to $x$. The degree of a vertex $x$ is the size of $\Gamma(x)$. A graph is regular if every vertex has the same degree. The $i$-neighborhood $\Gamma_{i}(x)$ is the set of vertices at geodetic distance $i$ to $x$. A graph is distance regular (DR) if for every pair of vertices $u$ and $v$ at distance $i$ apart the quantities

$$
\begin{aligned}
& a_{i}=\left|\Gamma_{i+1}(u) \cap \Gamma(v)\right| \\
& c_{i}=\left|\Gamma_{i-1}(u) \cap \Gamma(v)\right|
\end{aligned}
$$

solely depend on $i$ and not on the special choice of the pair $(u, v)$. In that situation the graphs $\Gamma_{i}$ are regular of degree $v_{i}$ and we will refer to the $v_{i}$ s as the successive degrees of $\Gamma$. A DR graph of diameter 2 is called a Strongly Regular Graph (SRG). Its parameters $(\nu, \kappa, \lambda, \mu)$ denote the number of vertices, the degree, the number of common neighbors of a pair of connected vertices, the number of common neighbors of a pair of disconnected vertices. The spectrum of a graph is the set of distinct eigenvalues of its adjacency matrix. It is denoted by $\left\{\lambda_{1}^{m_{1}}, \lambda_{2}^{m_{2}}, \ldots\right\}$, where $m_{i}$ stands for the multiplicity of the eigenvalue $\lambda_{i}$. 


\subsection{Linear codes}

A linear $q$-ary code of length $n$, dimension $k$, minimum distance $d$ is called an $[n, k, d]_{q}$, or an $[n, k]_{q}$ code for short. The (Hamming) weight of $x \in \mathbb{F}_{q}^{n}$ is the number of indices $i$ where $x_{i} \neq 0$. A two-weight code is an $[n, k]_{q}$ linear code having two nonzero weights $w_{1}$ and $w_{2}$. A three-weight code or 3 -weight code is an $[n, k]_{q}$ linear code having three nonzero weights $w_{1}<w_{2}<w_{3}$. In general, the weight distribution of the code is written in compact form as $\left[\left\langle i, A_{i}\right\rangle\right]$ where $i$ runs over the weights in the code, and $A_{i}$ is the number of codewords of weight $i$. The dual weight distribution is the weight distribution of the dual code. The duality is understood with respect to the standard inner product. The external distance of a linear code is the number of nonzero weights in its dual. A coset of a linear code $C$ is any translate of $C$ by a constant vector. A coset leader is any coset element that minimizes the weight. The weight of a coset is the weight of any of its leaders. The covering radius is the maximum weight of a coset. The coset graph $\Gamma_{C}$ of a code $C$ is defined on the cosets of $C$, two cosets being connected if they differ by a coset of weight one. Thus the diameter of this graph equals the covering radius of the code. A code is completely regular if the weight distribution of each of its cosets only depend on the weight of its coset leader. It is known [1] that a code is completely regular iff its coset graph is a DR graph. In that case its covering radius equals its dual distance.

From [1, Theorem 1.11.1], we have the following fact.

Theorem 1 If $C$ is a q-ary code, of minimum distance at least three, with dual weight distribution $\left[\left\langle i, A_{i}\right\rangle\right]$, then the spectrum of $\Gamma_{C}$ is $\left\{(n(q-1)-q i)^{A_{i}}\right\}$. Thus $A_{i}$ is the frequency of weight $i$ in $C^{\perp}$ and the multiplicity of the eigenvalue $n(q-1)-q i$.

A code is projective if the minimum distance of its dual code is at least three. The coset graph with $b$ loops $\Gamma_{C}^{b}$ of a code $C$ is obtained from $\Gamma_{C}$ by adding $b$ loops around every vertex. The following result is immediate from the previous theorem.

Corollary 1 If $C$ is a q-ary code, of minimum distance at least three, with dual weight distribution $\left[\left\langle i, A_{i}\right\rangle\right]$, then the spectrum of $\Gamma_{C}^{b}$ is $\left\{(b+n(q-1)-q i)^{A_{i}}\right\}$. Thus $A_{i}$ is the frequency of weight $i$ in $C^{\perp}$ and the multiplicity of the eigenvalue $b+n(q-1)-q i$.

\section{Main results}

If $\Omega \subseteq \mathbb{F}_{q}^{k}$ and $0 \notin \Omega$, we denote by $C(\Omega)$ the projective code of length $n=\frac{|\Omega|}{q-1}$ obtained as the kernel of the $k \times n$ matrix $H$ with columns the projectively non equivalent non zero elements of $\Omega$. Thus $H$ is the check matrix of $C(\Omega)$. To simplify notation, let $\Gamma_{C(\Omega)}=\Gamma(\Omega)$ and $\Gamma_{C(\Omega)}^{b}=\Gamma(\Omega)^{b}$.

The following result is immediate from the definitions in the Introduction, and will be used repeatedly and implicitly in the rest of the paper.

Theorem 2 The following are equivalent

- $\Omega($ resp $\Omega \cup 0)$ is an s-sum set,

- $\Gamma(\Omega)\left(\operatorname{resp.} \Gamma(\Omega)^{1}\right)$ is an $s-S W R G$.

Proof Observe that $\Gamma(\Omega)$ is a Cayley graph on the group $\left(\mathbb{F}_{q}^{k},+\right)$ with generators the columns of $H$. Thus the definition of an $s$-sum set can be regarded as a statement on the number of paths of length $s$ between 0 and $h$. 
The following result comes from the well-known connection between SRGs and twoweight codes [1,2]. It was observed implicitly in [4].

Theorem 3 If $C(\Omega)^{\perp}$ is a two-weight code, then both $\Omega \bigcup 0$ and $\Omega$ are s-sum sets for all $s>1$.

Proof If $C(\Omega)^{\perp}$ is a two-weight code, it is well-known that $\Gamma(\Omega)$ is an SRG [1,2]. The result follows by Theorem 2, and the observation, made in the Introduction, that SRGs are $s$-SWRG for all $s>1$.

The following result was observed in [12, Theorem III.6].

Theorem 4 If $\Omega$ or $\Omega \bigcup 0$ is an s-sum sets, then $C(\Omega)^{\perp}$ has at most three non-zero weights. Furthermore, if $s$ is even, then $C(\Omega)^{\perp}$ is an s-weight code.

We come to the coding-theoretic characterization of 3-sum sets which is contained in a slightly less detailed form in [4, Theorem 2.1]. The consideration of coset graphs with loops might seem artificial but is justified by the existence of several non trivial examples, as evidenced by Sect. 4 .

Theorem 5 Assume that $C(\Omega)^{\perp}$ is of length $n$ and has three nonzero weights $w_{1}<w_{2}<w_{3}$. Let $b$ be any integer.

- $\Omega$ is a 3-sum set iff $w_{1}+w_{2}+w_{3}=\frac{3 n(q-1)}{q}$;

- $\Omega \bigcup 0$ is a TSS iff $w_{1}+w_{2}+w_{3}=\frac{3(1+n(q-1))}{q}$;

- $\Gamma_{C(\Omega)}^{b}$ is a 3-SWRG iff $w_{1}+w_{2}+w_{3}=\frac{3(b+n(q-1))}{q}$.

Proof Follows by [20] Proposition 4.1, upon using either Theorem 1 or its corollary.

Remark In general, the parameters $\lambda, \mu, v$ of a 3-SWRG graph are related to the three nontrivial eigenvalues of the graph by the system of three equations in three unknowns given by [20, Prop. 3.1]. The resulting formulas are too messy to be displayed.

Example 1 If $q=2$ and $n=8$, then the possible triples of weights are $\{345,246,156,237$, $147,138\}$.

Example 2 If $C(\Omega)$ is the perfect binary Golay code, its dual weights are 8, 12, 16, and the second condition of the above theorem is satisfied

$$
8+12+16=\frac{3(23+1)}{2} .
$$

Example 3 If $C(\Omega)$ is the extended ternary Golay code, its coset graph is distance regular [1]. Still, since its dual weights are $8,12,16$, neither condition is satisfied, even though $\Gamma(\Omega)$ is DR. This is a case where DR does not imply SWRG.

The following result is new in the context of $s$-sum sets.

Theorem 6 Assume that $C(\Omega)^{\perp}$ is of length $n$ and has three nonzero weights $w_{1}<w_{2}=$ $\frac{n(q-1)}{q}<w_{3}$, with $w_{1}+w_{3}=\frac{2 n(q-1)}{q}$. Then $\Omega$ is an s-sum set for all odd $s>1$.

Proof Follows by [20] Proposition 4.2, upon using Theorem 1. 
The following result improves Prop. 4.3 of [11] by giving a simpler and more explicit diophantine equation bearing on the weights of $C(\Omega)^{\perp}$.

Theorem 7 Assume that $C(\Omega)^{\perp}$ is of length $n$ and has three nonzero weights $w_{1}<w_{2}<w_{3}$. Let $b$ be any integer.

- $\Omega$ is an s-sum set iff $\left(w_{3}-w_{2}\right)\left(n(q-1)-q w_{1}\right)^{s}+\left(w_{1}-w_{3}\right)\left(n(q-1)-q w_{2}\right)^{s}+$ $\left(w_{2}-w_{1}\right)\left(n(q-1)-q w_{3}\right)^{s}=0$.

- $\Omega \bigcup 0$ is an s-sum set iff $\left(w_{3}-w_{2}\right)\left(1+n(q-1)-q w_{1}\right)^{s}+\left(w_{1}-w_{3}\right)(1+n(q-1)-$ $\left.q w_{2}\right)^{s}+\left(w_{2}-w_{1}\right)\left(1+n(q-1)-q w_{2}\right)^{s}=0$.

- $\Gamma_{C(\Omega)}^{b}$ is an $s-S W R G$ iff $\left(w_{3}-w_{2}\right)\left(b+n(q-1)-q w_{1}\right)^{s}+\left(w_{1}-w_{3}\right)(b+n(q-1)-$ $\left.q w_{2}\right)^{s}+\left(w_{2}-w_{1}\right)\left(b+n(q-1)-q w_{2}\right)^{s}=0$.

Proof Follows by [20] Proposition 4.3, upon using Theorem 1 or its corollary.

Example 4 If $n=24, q=2$ the only triples $\left(w_{1}, w_{2}, w_{3}\right)$ satisfying the first condition of the above theorem for $s=5$ are those satisfying the conditions of Theorem 6 , namely $\{11+i, 12,13+i\}$, for $i=0,1, \ldots, 10$.

Motivated by many similar examples, we are led to the following conjecture.

Conjecture $\Omega$ is an $s$-sum set for some $s>3$ iff

$$
\left(w_{1}, w_{2}, w_{3}\right)=\left(w_{1}, \frac{n(q-1)}{q}, \frac{2 n(q-1)}{q}-w_{1}\right),
$$

for some $0<w_{1}<\frac{n(q-1)}{q}$. In other words, the only $s$-sum sets for $s>3$ are those satisfying the hypotheses of Theorem 6.

This is consistent with the observation in [20, p. 808]: "It remains open whether there are $\ell$-SWRG with $\ell>3$ that are not $m$-SWRG for all odd $m \neq \ell$ ". The next result shows that, when the conditions of Theorem 6 are not satisfied, a given code can yield an $s$-sum set for at most one value of $s$.

Theorem 8 Assume that $C(\Omega)^{\perp}$ is of length $n$ and has three nonzero weights $w_{1}<w_{2}<w_{3}$. If $w_{2} \neq \frac{n(q-1)}{q}$, or $w_{1}+w_{3} \neq \frac{2 n(q-1)}{q}$, then there is at most one $s>1$ such that $\Omega$ is an s-sum set.

Proof Follows by [20, Theorem 4.4], upon using Theorem 1 or its corollary.

\section{Classification in short lengths}

In this section we classify three-weight codes satisfying Theorem 6 for $q=2,3,4$, and $n$ small. For a 3-weight code $C$ of parameters $[n, k]_{q}$ with weights $w_{1}<w_{2}<w_{3}$, dual distance $\geq 3$, and respective frequencies $A_{1}=A_{w_{1}}, A_{2}=A_{w_{2}}, A_{3}=A_{w_{3}}$, denoted here by $\left[A_{1}, A_{2}, A_{3}\right]$, we recall the Pless power moments (PPM) [13].

$$
\begin{aligned}
A_{1}+A_{2}+A_{3} & =q^{k}-1 \\
A_{1} w_{1}+A_{2} w_{2}+A_{3} w_{3} & =n(q-1) q^{k-1} \\
A_{1} w_{1}^{2}+A_{2} w_{2}^{2}+A_{3} w_{3}^{2} & =n(q-1)(1+n(q-1)) q^{k-2} .
\end{aligned}
$$


The dimension of $C$ is constrained by the fact that the distance of $C^{\perp}$ is $\geq 3$. This gives an upper bound on $n-k$ by the tables of [10], and thus, a lower bound on $k$. The Delsarte bound [13, Theorem 11.3.3] states that the covering radius of a code is at most its external distance. Since $C$ is a 3-weight code, the covering radius of $C^{\perp}$ is $\leq 3$. This implies by the sphere-covering bound [5, p. 18] that

$$
q^{k} \leq 1+n(q-1)+\left(\begin{array}{l}
n \\
2
\end{array}\right)(q-1)^{2}+\left(\begin{array}{l}
n \\
3
\end{array}\right)(q-1)^{3} .
$$

When $q=2$, we can also use the tables on $t[N, K]$ the smallest covering radius of an $[N, K]_{2}$ code [5, p. 191]. This provides an upper bound on $k$. We denote by $B K L C(G F(q), n, k)$ the code given by Magma [15], as an $[n, k, d]_{q}$ with the largest $d$.

\subsection{Binary codes}

Observe first that, from the expression for $w_{2}$, the length $n$ must be even.

- If $n=4$ or $n=6$ the PPM eliminate all sets of parameters, returning non integral or non positive solutions.

- If $n=8, q=2$ the only triples $\left(w_{1}, w_{2}, w_{3}\right)$ satisfying the first condition of the above theorem for $s=5$ are $\{(1,4,7),(2,4,6),(3,4,5)\}$.

- It is easy to see that 1, 4, 7 cannot be the three weights of a binary code since $4-1=3$, and $4+1=5$.

- It can be proved, using Pless power moments, and MacWilliams transform, that there are exactly two codes of length 8 , distance $\leq 3$, and dual weights $(2,4,6)$, a [8, 2, 3] and a $[8,3,3]$ with respective weight distributions

$$
[\langle 0,1\rangle,\langle 3,1\rangle,\langle 5,1\rangle,\langle 8,1\rangle],
$$

and

$$
[\langle 0,1\rangle,\langle 3,2\rangle,\langle 4,2\rangle,\langle 5,2\rangle,\langle 8,1\rangle]
$$

- The Pless power moments return negative frequencies for a 3-weight codes with weights $(3,4,5)$ and dual distance $\geq 3$.

- If $n=10$, the PPM eliminate all parameters.

- If $n=12$, similar arguments as for $n=8$ only allows the weights $(5,6,7)$ and $(4,6,8)$. The first set admits the unique frequencies $(3,3,9)$. This is impossible since we must have $k \geq 5$. The second set admits eight possible triples of frequencies which reduce to 4 after using $5 \leq k \leq 8$. These triples are

$$
[16,12,3],[26,24,13],[42,40,45],[90,72,93] \text {. }
$$

The last two can be eliminated by MacWilliams transform. There are two codes with

$$
[\langle 0,1\rangle,\langle 4,16\rangle,\langle 6,12\rangle,\langle 8,3\rangle]
$$

or

$$
[\langle 0,1\rangle,\langle 4,26\rangle,\langle 6,24\rangle,\langle 8,13\rangle]
$$


These are $B K L C(G F(2), 12,5)$ and $B K L C(G F(2), 12,6)$ respectively. We do not know if they are unique with these weight distributions.

\subsection{Ternary codes}

Observe first that, from the expression for $w_{2}$, the length $n$ must be a multiple of 3 . Note also that a ternary code can only have even frequencies since the map $x \mapsto-x$ is a Hamming isomorphism.

- If $n=3$, we obtain the solution $(1,2,3)$ for the weights, and $[6,12,8]$ for the frequencies, which determines uniquely the universe code $\mathbb{F}_{3}^{3}$.

- If $n=6$, the only admissible triple of weights is $(2,4,6)$ which is impossible by the PPM.

- If $n=9$, the only admissible triple of weights are $(3,6,9),(4,6,8),(5,6,7)$. Observing that $3 \leq k \leq 6$, the possible triple of frequencies given by the PPM are, respectively,

- $[6,66,8],[24,192,26]$ and $[78,570,80]$. The last two can be ruled out by MacWilliams transform. We leave the construction of a ternary 3 -weight code with weight distribution $[\langle 0,1\rangle,\langle 3,6\rangle,\langle 6,68\rangle,\langle 9,8\rangle]$ as an open problem.

- None that is integral.

- $[6,8,12]$. A code with the dual weight distribution is $B K L C(G F(3), 9,6)$ in Magma notation. This code has covering radius 2 , missing the Delsarte bound by one. Hence it is not completely regular. So its coset graph is not DR, hence not a SRG, but it is an $s$-SWRG for all odd $s$ !

- If $n=12$, the only triple of weights not eliminated by PPM is $(6,8,10)$. We know that $3 \leq k \leq 6$. The only admissible triple of frequencies compatible with this condition are [17, 42, 21], [71, 96, 75] and [233, 258, 237] which all contain odd components. No code for $n=12$.

\subsection{Quaternary codes}

Observe first that, from the expression for $w_{2}$, the length $n$ must be a multiple of 4 .

- If $n=4$, there is no possible triple of weights.

- If $n=8$, the possible triples of weights are $(4,6,8)$ and $(5,6,7)$. The respective triple of frequencies are $[27,3,33]$, in the latter case, and

$$
[6,48,9],[42,168,45],[186,648,189]
$$

in the former, using the conditions $3 \leq k \leq 5$. The triple [27,3,33], leads to a MacWilliams transform with negative coefficients. A similar phenomenon happens for $[186,648,189]$. The triple of frequencies $[42,168,45]$ is realized for the binary first order Reed-Mueller code $R M(1,3)$ read over $\mathbb{F}_{4}$. The existence of an $[8,3]_{4}$ with weight distribution

$$
[\langle 0,1\rangle,\langle 4,6\rangle,\langle 6,48\rangle,\langle 8,9\rangle],
$$

is left as an open problem. 


\section{Infinite families}

\subsection{Binary cyclic codes}

There are many examples from binary cyclic codes. If we consider the codes of length $n=2^{m}-1$ with either the weights in [9, Tables 1, 2], that is either $\left\{2^{m-1}, 2^{m-1} \pm 2^{m-1-\ell}\right\}$, or $\left\{2^{m-1}, 2^{m-1} \pm 2^{(m-2) / 2}\right\}$, we see that the second condition of Theorem 5 is satisfied. These codes are cyclic codes with two zeros, closely related to preferred pairs of $m$-sequences [17].

\section{2 q-ary cyclic codes}

If we consider the codes $C^{\perp}$ of length $n=q^{m}-1$ with the weights in [9, Tables 6-8] they satisfy the relation

$$
w_{1}+w_{2}+w_{3}=3(1-1 / q)(n+1) .
$$

This shows, upon applying the third condition of Theorem 5 that the coset graph $\Gamma_{C}^{q-1}$ with $q-1$ loops is a $3-\mathrm{SWRG}$.

\subsection{Codes from Boolean functions}

In [16] it is proved that so-called plateaued Boolean functions $f$ on $\mathbb{F}_{2}^{n}$ have a Cayley graph $G_{f}$ on the group $\left(\mathbb{F}_{2}^{n},+\right)$ with generating set the support of $f$ that is

$$
\Omega_{f}=\left\{x \in \mathbb{F}_{2}^{n} \mid f(x)=1\right\},
$$

that is 3-SWRG and $\ell$-SWRG for all odd $\ell$ [16, Theorem 2]. Actually, this is the situation of Theorem 6. Thus, $\Omega_{f}$ is a TSS, and an $\ell$-sum set for all odd $\ell$. Moreover, $C\left(\Omega_{f}\right)$ is a three weight code of length $\left|\Omega_{f}\right|$ and codimension $n$. In that case the weights of $C\left(\Omega_{f}\right)$ are $\left\{\frac{n}{2}, \frac{n \pm 2^{(n+s-2) / 2}}{2}\right\}$, where $s$ is a parameter depending on $f$. These codes were introduced first in [7].

\subsection{Trace codes over fields}

The binary codes $C^{\perp}$ of length $n=2^{m-1}-1$ with the weights in Table 1 of [8], satisfy the relation

$$
w_{1}+w_{2}+w_{3}=3(1-1 / 2)(n+1) .
$$

This shows, by the third condition of Theorem 5, that the coset graph $\Gamma_{C}^{1}$ is a 3-SWRG. It also shows, by the second condition of Theorem 5, that the corresponding $\Omega$ with 0 adjoined is TSS. The $p$-ary codes $C^{\perp}$ of length $n=p^{m-1}-1$ with the weights in [21, Tables 3-4] satisfy the relation

$$
w_{1}+w_{2}+w_{3}=3(1-1 / p)(n+1)
$$

This shows, by the third condition of Theorem 5 , that the coset graph $\Gamma_{C}^{p-1}$ with $p-1$ loops is a $3-S W R G$. 
Table 1 Constructions from codes

\begin{tabular}{lllll}
\hline$n$ & $q$ & $\ell$ & Graph & References \\
\hline $2^{m}-1$ & 2 & 3 & $\Gamma_{C}^{1}$ & {$[9]$} \\
$q^{m}-1$ & $q$ & 3 & $\Gamma_{C}^{q-1}$ & {$[9]$} \\
$2^{m}$ & 2 & Odd & $\Gamma_{C}$ & {$[16]$} \\
$2^{m-1}-1$ & 2 & 3 & $\Gamma_{C}^{1}$ & {$[8]$} \\
$p^{2 m}-p^{m}$ & $p$ & 3 & $\Gamma_{C}$ & {$[18]$} \\
\hline
\end{tabular}

\subsection{Trace codes over rings}

Trace codes over rings can give few-weight codes over fields by application of a Gray map. The three-weight $p$-ary codes $C^{\perp}$ of length $n=p^{2 m}-p^{m}$ in [18] satisfy the relation

$$
w_{1}+w_{2}+w_{3}=3(1-1 / p) n .
$$

This shows, by the first condition of Theorem 5, that the coset graph $\Gamma_{C}$ is a 3-SWRG, or, equivalently, that the $\Omega$ such that $C(\Omega)=C$ is a TSS.

\subsection{Summary}

We summarize the previous subsections in the following table.

\section{Conclusion and open problems}

In this article, we have developed the dictionary between multiple sum sets and strongly walk-regular graphs via the coset graph of the code attached to such a set. Motivated by concrete examples we have considered coset graphs with loops. In comparison with the classical works on the topic $[4,11,12]$ our note contains many infinite families of such sets or graphs. It seems that most known families of three-weight codes were constructed in the last five years. Classification in short length has been made for $q=2,3$, 4. It might be of interest to go for larger fields or longer lengths.

One aim of research would be to find more three-weight codes satisfying the first condition of Theorem 5. Only one infinite family can be found in $\$ 4$. Another open problem would be to characterize by their parameters three-weight codes the coset graph of the dual code of which is both DR and SWRG. Example 3 shows that this is not always true. Eventually, the main open problem is the Conjecture of Sect. 3, which can be summarized as follows: an $s$-sum set for some $s>3$ is an $s$-sum set for all odd $s>3$.

\section{References}

1. Brouwer A.E., Cohen A.M., Neumaier A.: Distance-Regular Graphs. Springer, Berlin (1989). https://doi. org/10.1007/978-3-642-74341-2.

2. Brouwer A.E., Haemers W.H.: Spectra of Graphs. Springer, New York (2012). https://doi.org/10.1007/ 978-1-4614-1939-6.

3. Calderbank R., Kantor W.M.: The geometry of two-weight codes. Bull. Lond. Math. Soc. 18, 97-122 (1986). 
4. Courteau B., Wolfmann J.: On triple sum sets and two or three-weight codes. Discret. Math. 50(2-3), 179-191 (1984).

5. Cohen G.D., Honkala I., Litsyn S., Lobstein A.: Covering Codes. North-Holland, Amsterdam (1997).

6. Delsarte P.: Weights of linear codes and strongly regular normed spaces. Discret. Math. 3(1), 47-64 (1972).

7. Ding C.: Linear codes from some 2-designs. IEEE Trans. Inf. Theory 61(6), 3265-3275 (2015).

8. Ding K., Ding C.: Binary linear codes with three weight. IEEE Commun. Lett. 18(11), 1879-1882 (2014).

9. Ding C., Li C., Li N., Zhou : Three-weight cyclic codes and their weight distributions. Discret. Math. 339(2), 415-427 (2016).

10. Grassl, M.: www.codetables.de

11. Griera M.: On $s$-sum sets and three weight projective codes. Springer Lect. Notes Comput. Sci. 307, 68-76 (1986).

12. Griera M., Rifa J., Hughet L.: On $s$-sum sets and projective codes. Springer Lect. Notes Comput. Sci. 229, 135-142 (1986).

13. Huffman W.C., Pless V.: Fundamentals of Error Correcting Codes. Cambridge University Press, Cambridge (2003).

14. Ma S.L.: A survey of partial differences sets. Des. Codes Cryptogr. 4(4), 221-261 (1994).

15. Magma website http://magma.maths.usyd.edu.au/magma/

16. Riera C., Solé P., Stanica P.: A complete characterization of plateaued Boolean functions in terms of their Cayley graph. Springer Lect. Notes Comput. Sci. 10831, 1-8 (2018).

17. Sarwate D.V., Pursley M.B.: Crosscorrelation properties of pseudorandom and related sequences. Proc. IEEE 68(5), 593-619 (1980).

18. Shi M., Rongsheng W., Liu Y., Solé P.: Two and three weight codes over $\mathbb{F}_{p}+u \mathbb{F}_{p}$. Cryptogr. Commun. 9(5), 637-646 (2017).

19. Shi M., Sepasdar Z., Alahmadi A., Solé P.: On two weight $\mathbb{Z}_{2}^{k}$-codes. Des. Codes Cryptogr. 86, 1201-1209 (2018).

20. van Dam E.R., Omidi G.R.: Strongly walk-regular graphs. J. Comb. Theory A 120, 803-810 (2013).

21. Yang S., Yao Z.-A.: Complete weight enumerator of a family of three-weight linear codes. Des. Codes Cryptogr. 82(3), 1-12 (2017).

Publisher's Note Springer Nature remains neutral with regard to jurisdictional claims in published maps and institutional affiliations. 